Jap. J. Physiol., 24, 587-603, 1974

\title{
EFFECTS OF OSMOLARITY CHANGE ON THE EXCITATION-CONTRACTION COUPLING OF BULLFROG VENTRICLE
}

\author{
Hiroshi Kawata, Kimie Kawagoe, and Iwao Tateyama* \\ Department of Physiology, Fukuoka University \\ *Department of Physiology, Kyushu University, Fukuoka, Japan
}

\begin{abstract}
Summary The effect of hyper- and hypotonic solutions on the electrical as well as the mechanical activities of the bullfrog ventricle were investigated.

Hypertonic solutions up to $400 \%$ tonicity, made by adding sucrose, showed two kinds of inhibitory effect; i.e. a consistent, rapid shortening of the action potential duration and a slowly progressing suppression of contractile tension. The inhibitory effect of the hypertonic solution up to $300 \%$, made by adding $\mathrm{NaCl}$, was more pronounced on the contractile process than on the electrical phenomena. In this condition, the inhibition of the twitch tension occurred with two time constants. The first, rapid decline seems to be related to the suppressive effect on some superficial site while the slower decreasing phase showed a similar time course as the development of contracture.

Hypotonic $(33.3 \%$ and $12.5 \%$ ) perfusion revealed triphasic changes in the time course of single twitch without accompanying any parallel change in the action potential.

From these results it was concluded that the bullfrog ventricular muscle has some superficial site for $\mathrm{Ca}$ regulation which is very sensitive to osmolarity change.
\end{abstract}

It is known that the twitch contraction of skeletal muscle can be strongly suppressed in hypertonic media without any essential change in the action potential (HodgKin and Horowicz, 1957; HowarTH, 1958). It has been shown that the caffeine-induced contracture is also inhibited by hypertonic perfusion (IsAACSON, 1969). The mechanism of the contractile inhibition by hypertonicity is still unknown although a direct suppressive effect on the contractile elements is postulated by many authors.

Recently LÄNNERGREN and NOTH (1973) have reported that the hypertonic

Received for publication May 9, 1974

河田 溥, 川越紀美江，立山 蟋 
medium itself can elicit a phasic type of contraction in the isolated single muscle fiber and that this contraction is attributed to the activation of sarcoplasmic reticulum. Thus, in the skeletal muscle the hypertonicity has two different types of effect on the contractile process, i.e. an inhibitory effect on the myofilaments and an activating effect on the excitation-contraction (E-C) coupling. On the other hand, the effect of hypertonicity on the heart muscle has not yet been fully analyzed. Although some investigations (SPERELAKis et al., 1960; KAWATA, 1961; Little and Sleator, 1969; HermsmeYer et al., 1972) have shown that the hypertonic media shorten the cardiac action potential, there are few investigations on the effects of tonicity on myocardial contractility (KOCH-WESER, 1963; LITTLE and SLEATOR, 1969). The present study is aimed at clarifying the effect of hypertonicity as well as hypotonicity both on the electrical and mechanical phenomena in the frog ventricular muscle.

\section{METHODS}

Small strips obtained from the bullfrog (Rana catesbiana) ventricle were used throughout the experiments. After a thin ring-formed strip had been dissected from the middle one-third of the ventricular wall, the preparation was cut along the heart axis and mounted in a lucite chamber. The lengthened muscle strip was then partitioned into three compartments by using two thin celluloid plates and vaseline. The middle compartment, $5 \mathrm{~mm}$ wide, was perfused with isotonic sucrose solution while the two side compartments were perfused with normal Ringer solution (perfusion rate 3 to $4 \mathrm{ml} / \mathrm{min}$ ).

The muscle length in the measuring side compartment was 2 to $3 \mathrm{~mm}$. The transmembrane potential and isometric tension were simultaneously recorded in the measuring compartment (contents $2 \mathrm{ml}$ ). After a rest perfusion period of $30 \mathrm{~min}$ the muscle preparation was stimulated with short pulses $(10 \mathrm{msec})$ at a constant rate (usually 6 or 12 shocks $/ \mathrm{min}$ ) through a pair of $\mathrm{Ag}-\mathrm{AgCl}$ electrodes placed in both side compartments. In general, a period of $30 \mathrm{~min}$ or more was necessary until the twitch tension became steady after the start of driving. When the partition was satisfactory no contractile response could be observed in the middle compartment under the binocular microscope on the application of either depolarizing or hyperpolarizing pulses. The total current was monitored through a resistor.

The normal Ringer solution had the following composition in $\mathrm{mM}: \mathrm{NaCl}$ 111, $\mathrm{KCl} 2.7, \mathrm{CaCl}_{2} 2.4, \mathrm{NaHCO}_{3} 2.4, \mathrm{KH}_{2} \mathrm{PO}_{4}$ 0.37, and glucose 11.1; the $\mathrm{pH}$ of the fluid was 7.2. A hypertonic solution was made by adding either various amounts of sucrose (50 to $750 \mathrm{~mm}$ ) or $251.5 \mathrm{~mm}$ of $\mathrm{NaCl}$ (tonicity $300 \%$ ) to the normal Ringer's solution while a hypotonic solution was prepared by omitting either $83.9 \mathrm{~mm}$ of $\mathrm{NaCl}$ (tonicity $33.3 \%$, i.e. $1 / 3$ of normal) or $106 \mathrm{~mm}$ of $\mathrm{NaCl}$ $(12.5 \%$, i.e. $1 / 8$ of normal tonicity ) from the normal Ringer. The potassium 
contracture solution was prepared by simply replacing all the $\mathrm{NaCl}$ of the sucrosehypertonic solution with an equal amount of $\mathrm{KCl}$.

To check the sucrose insulation, a series of depolarizing and hyperpolarizing pulses were applied to both of the heart and skeletal toe muscles. The membrane potential was recorded from a muscle cell in the vicinity of the partition plate. In the case of skeletal muscle, a subthreshold depolarizing as well as a hyperpolarizing pulse produced electrotonic potential changes while only the suprathreshold depolarizing current pulse elicited active electrical and mechanical responses (see KAWATA and CHEN, 1972). On the contrary, the ventricular muscle generated an action potential even on applying a hyperpolarizing pulse (anodalmake response). Because such a response was never observed in the case of skeletal muscle, it could be attributed neither to the incomplete insulation in the middle compartment nor to the poor condition of the preparation, but rather to the structural complexity of the ventricular muscle as suggested by Goто and Brooks (1969). The difficulty in getting a spatial homogeneity of the potential distribution in the myocardium might be the main cause of undesirable depolarization which occurred in some cells of the measuring compartments. Nevertheless we used the short anodal pulses of $10 \mathrm{msec}$ duration as driving pulses throughout the experiment because this condition per se did not restrict the purpose of the present experiments. Furthermore, with such short pulses no fundamental differences in the configuration of the action potential as well as the twitch tension could be observed between cathodal and anodal stimulations.

A few experiments were done in order to check the effect of change in the Donnan equilibrium when the concentration of $\mathrm{NaCl}$ in the bathing medium was altered. In these experiments the $[\mathrm{K}]_{0} \cdot[\mathrm{Cl}]_{\mathrm{o}}$ product of the test solution was adjusted either to the isotonic $[\mathrm{K}]_{\mathrm{i}} \cdot[\mathrm{Cl}]_{\mathrm{i}}$ value, $363.8(\mathrm{mM})^{2}$ or to the steady state hyper- and hypotonic $[\mathrm{K}]_{\mathrm{i}} \cdot[\mathrm{Cl}]_{\mathrm{i}}$ value, $3274.2(\mathrm{mM})^{2}$ and $40.4(\mathrm{~mm})^{2}$, respectively, under the assumption that the heart muscle behaves as a perfect osmometer. It was found that the effect of change in $[\mathrm{K}]_{\mathrm{o}}$ on the twitch contraction is very large when the $[\mathrm{K}]_{\mathrm{o}} \cdot[\mathrm{Cl}]_{\mathrm{o}}$ was adjusted to $363.8(\mathrm{~mm})^{2}$, the isotonic value. On the other hand, when the value was adjusted to $3274.2(\mathrm{~mm})^{2}$ or $40.4(\mathrm{mM})^{2}$, the changes in the twitch tension caused both by hyper- and hypotonicities were essentially the

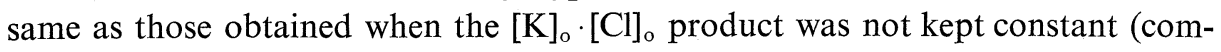
pare Fig. 8 with Fig. 10). Thus, in most of the experiments using $\mathrm{NaCl}$-hypertonic as well as hypotonic solutions, the $[\mathrm{K}]_{\circ} \cdot[\mathrm{Cl}]_{\text {。 }}$ product was not kept constant because this factor was not crucial for the present results.

All experiments were carried out at the room temperature $\left(20-22^{\circ} \mathrm{C}\right)$.

\section{RESULTS}

\section{Effects of sucrose-hypertonicity on electrical and mechanical phenomena}

Records A and B in Fig. 1 show changes in the twitch tension caused by a 
sucrose-hypertonic perfusion (tonicity $300 \%$ ). In Fig. 1A the initial 40 contractions during hypertonic perfusion were superimposed. It can be clearly seen that the maximum twitch tension is suppressed by hypertonic perfusion and that this is exclusively due to the shortening of the time to peak tension (TPT). The rate of rise of tension as well as the total contraction time remained practically unchanged while a slight elevation of the baseline (contracture) is noticeable in the figure. Upon returning the muscle to the normal Ringer solution the twitch tension was restored almost completely (Fig. 1B). The records were photographed and superimposed at intervals of $5 \mathrm{~min}$. It is also seen in the figure that a contracture of about $0.35 \mathrm{~g}$ appeared during the hypertonic perfusion and that the twitch contraction did not reappear until this contracture tension subsided below a certain level.

The time course of changes in the active and resting tensions observed during the hypertonic perfusion (tonicity $300 \%$ ) was summarized in Fig. 2 (open and solid circles) for five different muscle preparations in which the whole time course could be followed without being disturbed by a spontaneous excitation block. The twitch height immediately before hypertonic perfusion was expressed as $100 \%$. The graphical analysis revealed that the falling phase of twitch contraction consists of two phases whose rate constants are $0.40 \mathrm{~min}^{-1}$ and $0.07 \mathrm{~min}^{-1}$, respectively. The fast phase seems to account for the TPT-, therefore, action potential duration-dependent phase of tension decline whereas the slow phase seems to run parallel with the development of contracture.

Figure 3 shows changes both in the action potential and twitch contraction
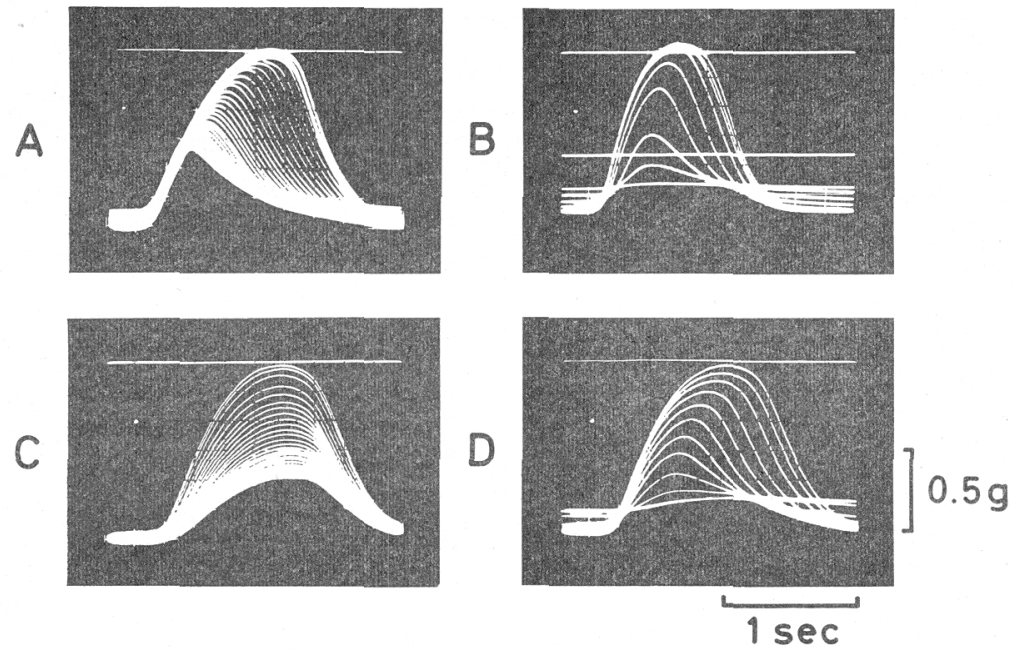

Fig. 1. Examples of the typical tension change caused by two kinds of hypertonicities. (A) and (B) show the effect of a solution made hypertonic (tonicity $300 \%$ ) by addition of sucrose while (C) and (D) show the effect of $\mathrm{NaCl}$-hypertonic solution of the same tonicity. (B) and (D) show the recovery process. Note the marked elevation of the resting tension in these records. 


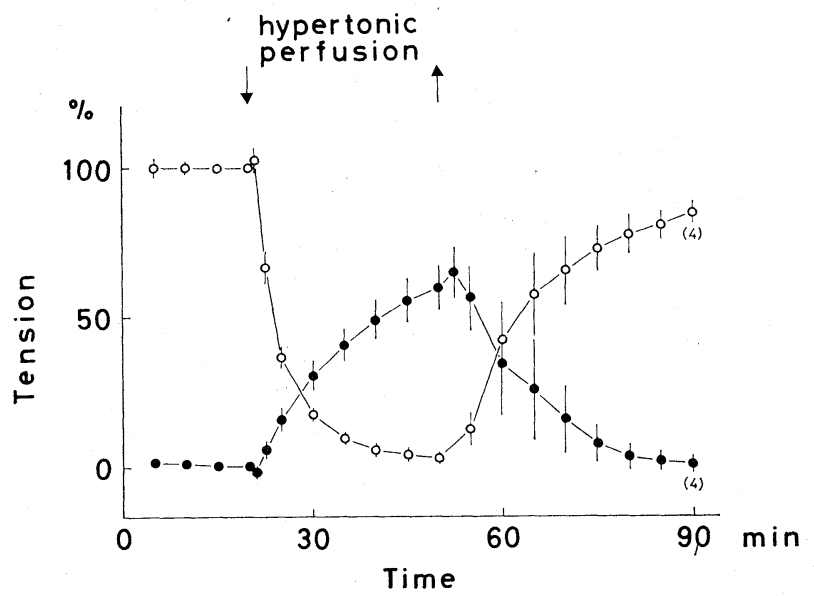

Fig. 2. Time course of the twitch inhibition (open circles) caused by sucrose-hypertonic solution (tonicity $300 \%$ ). The twitch height immediately before hypertonic perfusion was expressed as $100 \%$. The results obtained from five different muscles were summarized. Changes in the resting tension were also plotted (solid circles). Vertical bars indicate $\pm \mathrm{SE}$ of the mean.

when the tonicity of solution was raised by $100 \%$ (i.e. tonicity $200 \%$ ). In Fig. 3(a) the arrow shows where hypertonic perfusion began. The twitch decreases gradually. Accompanying this a gradual shortening of the action potential is seen in the figure (see also Fig. 3(b) B, C, D). Prior to this decline of contractile response it was noted that the threshold for excitation was elevated. This resulted in a failure both in the electrical and mechanical responses. This occurred again in the last part of the recording. Thus, the rise in the threshold, together with shortening in the action potential and fall in the peak tension, was consistently observed during hypertonic perfusion. Figure 3(a) are the photographical records. Records A, B, C, and D are the responses shown in Fig. 3(a). It is clearly seen that the shortening of the action potential occurs without any noticeable accompanying changes in the resting potential and the overshoot of the action potential. It should also be noted that the shortening of the action potential is exclusively due to the acceleration of phase 2 (plateau phase) and that the slope of phase 3, the final rapid repolarization, remains practically unchanged. Accompanying this, the twitch contraction decreases gradually without showing any change in the resting tension.

As stated above the elevation of the threshold for excitation was frequently observed during sucrose-hypertonic perfusion. In order to test this prolonged depolarizing as well as hyperpolarizing pulses were applied both in the normal and the hypertonic solutions. The results are shown in Fig. 4. In Fig. 4A four different depolarizing pulses of $1 \mathrm{sec}$ duration were applied. A subthreshold depolarization failed to elicit any active response. As the current intensity was 
(a)

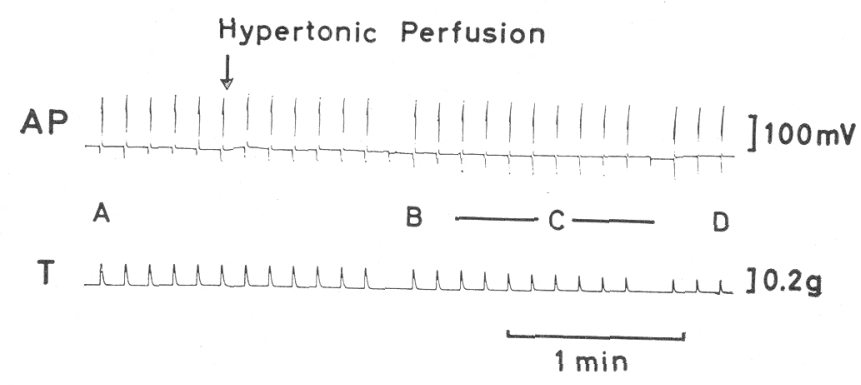

(b)
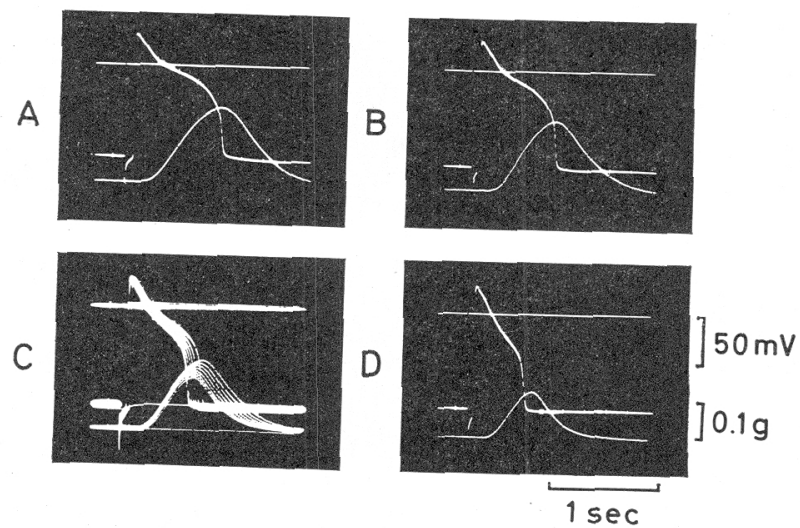

Fig. 3. Effects of sucrose-hypertonic solution (tonicity $200 \%$ ) on the action potential and twitch contraction of bullfrog ventricle. (a) is a paper record and shows a rapid decrease in the twitch height (b) illustrates the photographical records which were taken from the paper record (a). (A): control, (B), (C), and (D): during hypertonic perfusion.

increased the plateau level of the action potential was shifted towards more positive potential region. Accompanying with this the contractile tension was augmented in which both the rate of rise of tension and the time to peak tension were increased. Figure 4B shows the effects of three anodal pulses with different intensities. Records $\mathrm{C}$ and D of Fig. 4 show the responses obtained in the hypertonic medium (tonicity $200 \%$ ). It is seen that the membrane excitabililty was strongly suppressed. Only the strongest depolarizing pulse elicited a shortened action potential which was not followed by any mechanical response. Records $\mathrm{B}$ and $\mathrm{D}$ also show that the membrane resistance was not markedly changed in the hypertonic solution.

Further experiments were carried out in order to clarify whether the contractile machinery itself is also suppressed at the time when the twitch tension is inhibited by hypertonic perfusion. Figure 5 shows that a full-size contracture 

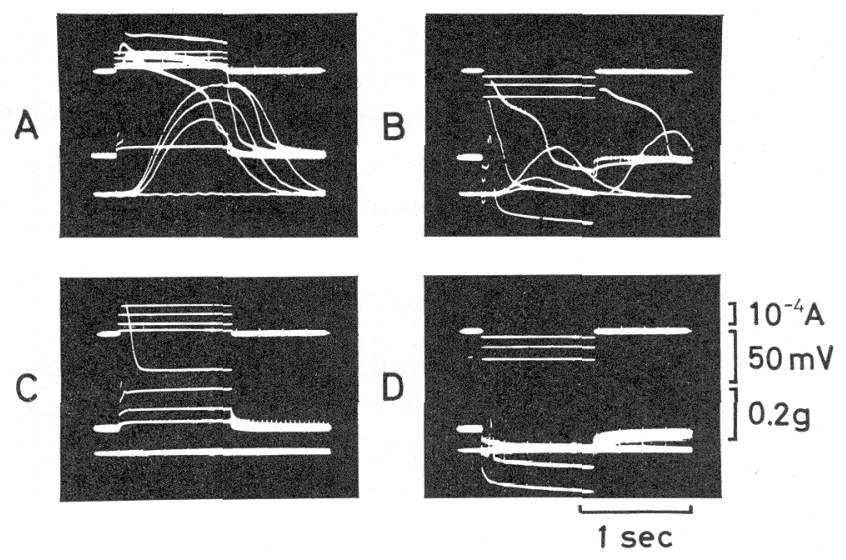

Fig. 4. Polarization experiments. (A) and (B) show the electrical and mechanical responses in normal Ringer while (C) and (D) show those in sucrose-hypertonic solution (tonicity $200 \%$ ). In each cases four different depolarizing (A, C) and three different hyperpolarizing $(\mathrm{B}, \mathrm{D})$ pulses of $1 \mathrm{sec}$ were applied.

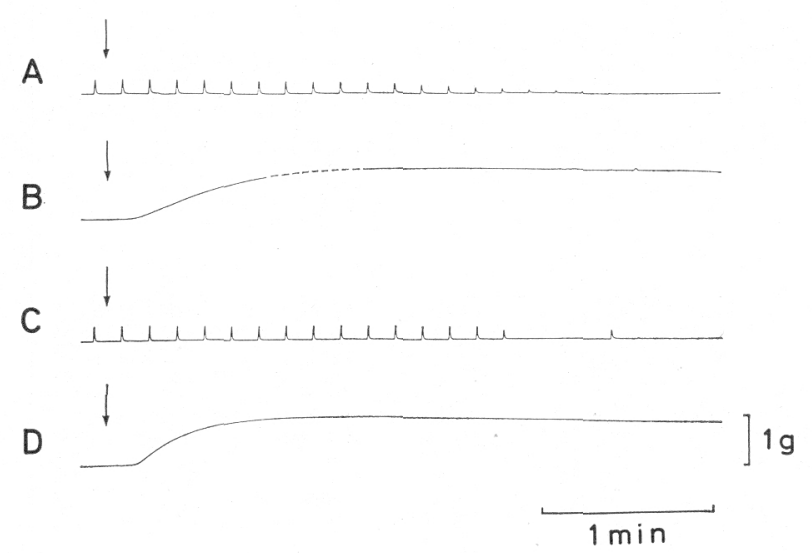

Fig. 5. Potassium-contracture experiments in sucrose-hypertonic media. The records from (A) to (D) show a successive series of the experiments on the same preparation. (A): A control twitch and effect of sucrose-hypertonic solution (tonicity $200 \%$ ). (B): Contracture induced by a sodium-free, high-potassium, hypertonic solution (tonicity $200 \%$ ). (C): A control twitch and effect of hypertonic solution (tonicity $300 \%$ ). (D): Contracture induced by a sodium-free, high-potassium, hypertonic solution (tonicity $300 \%$ ).

could be elicited by perfusion of hypertonic (300\%), high-potassium contracture solution (B) even after the twitch was completely suppressed by an ordinary hypertonic perfusion (A). Figure 5C and D show the effects of $400 \%$ tonicity. In this example, too, a powerful contracture could still be induced by high-potas- 
sium solution immediately after the twitch had been suppressed by the same hypertonicity. The magnitude of the potassium contracture tension did, however, decline as the interval between the onset of ordinary hypertonic and that of high-potassium hypertonic perfusions increases. Furthermore, the higher the tonicity was, the faster was the speed of this decline. Thus, the decline of the potassium contracture seems to be inversely related to the extent of development of hypertonic contracture.

\section{Effects of $\mathrm{NaCl}$ hypertonicity on the action potential and twitch contraction}

When the ventricular muscle preparation was perfused with a solution of $300 \%$ tonicity, made hypertonic by adding $\mathrm{NaCl}$ of $251.5 \mathrm{~mm}$ to normal Ringer solution, changes as illustrated in Fig. 6 were observed. Figure 6A is a control response in normal Ringer solution. In Fig. 6B a control response (1) and a response recorded $50 \mathrm{sec}$ after the onset of hypertonic perfusion (2) were superimposed. The shift of the baseline in the membrane potential is artificial so that one can compare them easily. It is clearly seen that the twitch contraction was suppressed without showing any noticeable changes in configuration of the action potential. Thus, the negative inotropic effect is solely due to a reduction in the rate of force development. The time to peak tension remained unchanged. Figure $6 \mathrm{C}$ was photographed about $15 \mathrm{~min}$ after the onset of hypertonic perfusion. The twitch tension was further suppressed while in this stage the action potential was shortened to about one-third of the control. Upon returning to the normal Ringer solution the electrical as well as the mechanical responses were restored towards control level (Fig. 6D).
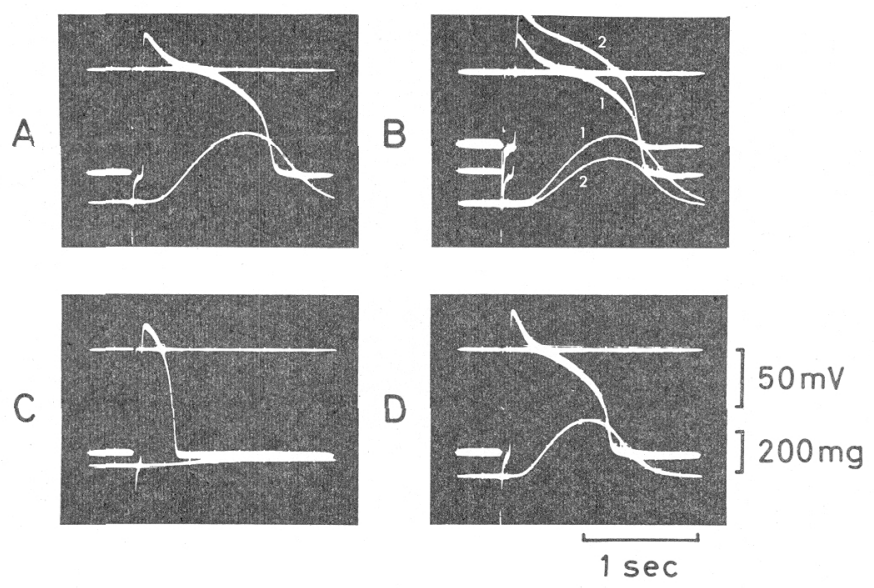

Fig. 6. Effect of NaCl-hypertonic solution (tonicity $300 \%$ ) on the action potential and twitch contraction. (A) and (D) are the responses of the control and recovery. Records (B) and $(\mathrm{C})$ are photographed $50 \mathrm{sec}$ and $15 \mathrm{~min}$ after the start of hypertonic perfusion, respectively. Note the temporal relationship between the shortened action potential and the slower uprising, weak contraction in (C). 
The pattern of change in the twitch contraction during $\mathrm{NaCl}$-hypertonic perfusion is also demonstrated in Fig. 1C and D. In Fig. 1C the first 40 contractions during hypertonic perfusion $(300 \%)$ were superimposed while in record D the last two in the hypertonic medium and 10 contractions during the recovery were photographed every $5 \mathrm{~min}$. As expected from the records shown in Fig. 6 the pattern of this change is quite different from that of sucrose hypertonicity. The decrease of contractile force is essentially due to the change in the rate of rise of tension development. A weak contracture also developed during the $\mathrm{NaCl}$ hypertonic perfusion (Fig. 1D).

Figure 7 summarizes the whole time course of the tension change during and after the hypertonic perfusion (tonicity $300 \%$ ) obtained from six different muscles. On exposing the muscle to hypertonic medium the twitch tension fell in two phases; rapid and slow. The rate constants for both phases measured graphically were $0.50 \mathrm{~min}^{-1}$ and $0.06 \mathrm{~min}^{-1}$, respectively. Changes in the action potential seem not to be essential for this process since the time to peak tension was never shortened during the hypertonic perfusion.

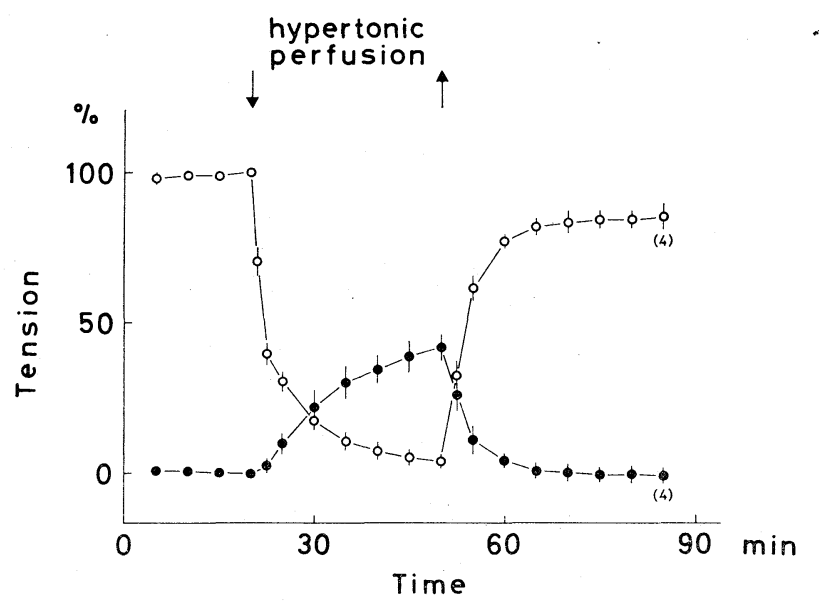

Fig. 7. Time course of the twitch inhibition (open circles) caused by $\mathrm{NaCl}$-hypertonic solution (tonicity $300 \%$ ). The twitch height immediately before hypertonic perfusion was expressed as $100 \%$. The results obtained from six different preparations were summarized. Changes in the resting tension were also plotted (solid circles). Vertical bars indicate $\pm \mathrm{SE}$ of the mean.

3. Effects of hypotonicity on the action potential and the twitch contraction

In comparison with the effect of hypertonicity the hypotonicity affects the twitch contraction in a more complicated manner. Figure 8 summarizes the results obtained from five different ventricular preparations. Application of hypotonic perfusion (tonicity $33.3 \%$ ) was made for $60 \mathrm{~min}$ in all examples. Similarly to Fig. 7 the changes were expressed as $\%$ of the control response immediately 


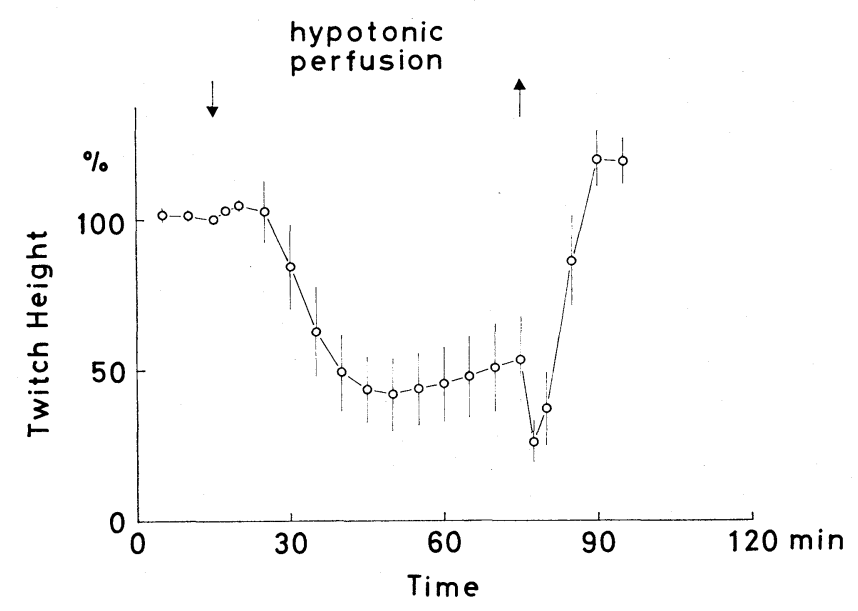

Fig. 8. Effect of hypotonic solution (tonicity $33.3 \%$ ) on the ventricular contraction. The results from five different frogs were summarized. Note that there is a six-phasic change in the twitch contraction during and after hypotonic perfusion for $60 \mathrm{~min}$. Vertical bars indicate $\pm \mathrm{SE}$ of the mean.

before the onset of hypotonic perfusion. In spite of considerable variation among preparations one can recognize that there are at least 6 phases in the time course of the tension change during hypotonic and recovery perfusion. They are 1 . initial increase, 2. subsequent large decrease, 3. delayed slower increase, 4. rapid fall on returning to normal Ringer solution, 5. subsequent rapid and large rise, and 6. final slow decrease towards the control level. The magnitude and the duration of the initial positive inotropism (phase 1) were variable and they seem to be reciprocally related to the extent of the subsequent decrease (phase 2). Usually the secondary rise (phase 3 ) took place about 30 min after the beginning of hypotonic perfusion.

The changes observed during recovery period are interesting. A sharp dip (phase 4 to phase 5) was observed in all preparations. This decrease in tension occurred within a relatively short time $(c a .2 .5 \mathrm{~min})$. It would be noteworthy that this period approximately coincides with that of the initial rapid fall during hypertonic perfusion (Fig. 7). Besides this undershoot-phenomenon there was an overshoot during the recovery. The twitch tension exceeded the control level $12 \mathrm{~min}$ after the onset of recovery perfusion. In general, the time course of the final recovery (phase 6) was very slow.

Quite a similar change was also observed when the tonicity of the bathing solution was lowered to $1 / 8$ of normal (tonicity $12.5 \%$ ). Figure 9 shows the effect of $1 / 8$ hypotonicity on the action potential and the twitch contraction. Record $\mathrm{A}$ is a control response and records $\mathrm{B}$ and $\mathrm{C}$ are the responses obtained in the hypotonic medium. The active twitch tension increases initially (B) and then 

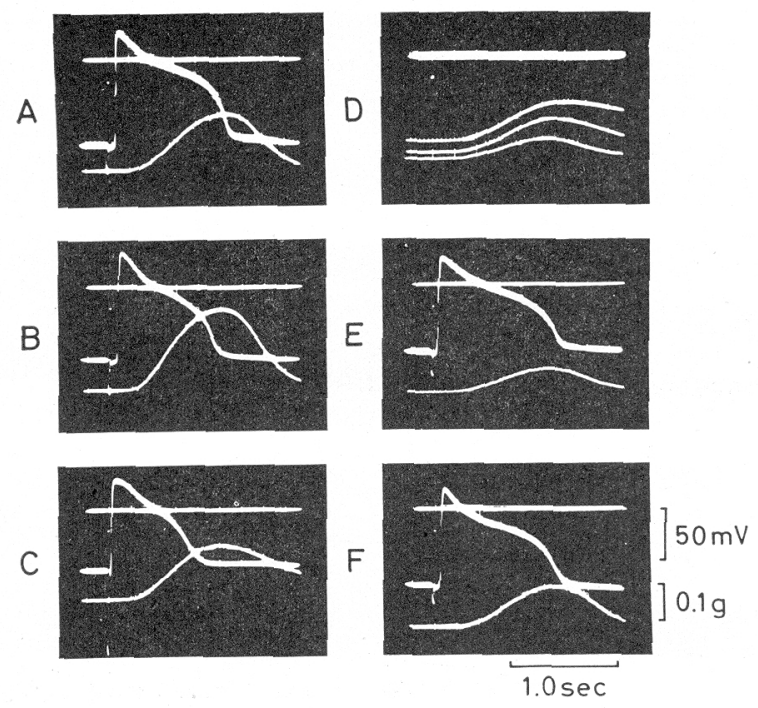

Fig. 9. Effect of hypotonic solution (tonicity $12.5 \%$ ) on the action potential and twitch contraction. (A): Control, (B), (C): During hypotonic perfusion, (D): Immediately after the onset of reperfusion of normal Ringer. (E), (F): Recovery in normal Ringer, 8 min and 55 min after the onset of perfusion, respectively.

decrease (C) without showing any change in the time to peak tension while the action potential shortens gradually. The resting membrane potential was reduced by about $25 \mathrm{mV}$ during hypotonic perfusion. The degree of the shortening of action potential in this medium was, however, not so large as in the hypertonic medium. On the other hand, a considerable contracture occurred especially in the latter half of the hypotonic perfusion (record $\mathrm{C}$ and the uppermost tension trace in record D). Thus, as seen in the figure, the total tension (resting tension + active tension) exceeds the twitch height of the control response though the active tension was considerably suppressed in this stage.

In Fig. 9D the perfusion was switched from a hypotonic to a normal solution. The uppermost twitch response was recorded immediately before the exchange of solution and it is essentially the same as the record C. The other two responses were the first and the second contractions in the normal Ringer solution. On returning to the normal Ringer solution a dramatic change was observed. The diastolic tension fell rapidly. This consequently caused a decrease in the active tension. At the same time, the rate of relaxation was augmented and this resulted in a shift of the time to forward peak tension. These changes in Fig. 9D corres pond to the change termed as phase 4 in Fig. 8. Records E and F of Fig. 9 are obtained $8 \mathrm{~min}$ and $55 \mathrm{~min}$, respectively, after returning to the normal Ringer. In such an extremely low tonicity as $12.5 \%$ the recovery process required more time than in the case shown in Fig. 8. It should be noted that the heart muscle 
was able to survive in such a low osmolarity medium. We have tested the effects of $1 / 8$ hypotonicity on the contractility in many preparations and confirmed that the muscle preparation has a surprisingly stable ability for restoration.
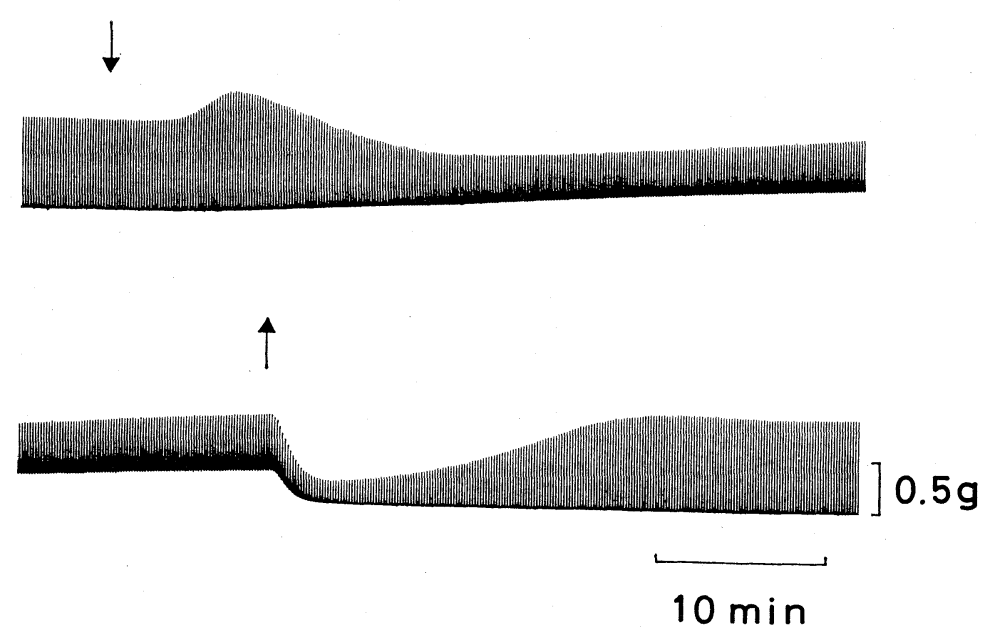

Fig. 10. Effect of hypotonic solution (tonicity $33.3 \%$ ) on the resting and active tension. The $[\mathrm{K}]_{\mathrm{o}} \cdot[\mathrm{Cl}]_{\mathrm{o}}$ product was adjusted so that the hypotonic $[\mathrm{K}]_{\mathrm{i}} \cdot[\mathrm{Cl}]_{\mathrm{i}}$ value of $40.4(\mathrm{mM})^{2}$ was kept constant. Note the marked elevation in the baseline and the slower relaxation rate of each contractions during hypotonic perfusion.

Figure 10 shows an example of the original tension record in which the hypotonic solution (tonicity $33.3 \%$ ) was perfused for $60 \mathrm{~min}$. Besides the changes in the active tension which is essentially the same as those shown in Fig. 8, a remarkable change in the resting tension is noticeable in the figure. The rise in the resting tension seems to occur in two ways, namely, a generation of the sustained contracture and a summation of twitch tensions due to the extremely slow relaxation in each contraction. The fall of the resting tension during recovery perfusion is very rapid as compared with its onset. The half times of the rising and falling phase are $28 \mathrm{~min}$ and $1.2 \mathrm{~min}$, respectively. In order to clarify this an isotonic low $\mathrm{Na}$ solution was applied after a 30 min-perfusion of hypotonic solution. The low $\mathrm{Na}$ solution was made by replacing $\mathrm{NaCl}$ in the normal Ringer solution with equimolar choline chloride containing $10^{-4} \mathrm{M} /$ liter atropine sulfate. The amount of $\mathrm{NaCl}$ removed was adjusted so that the remaining amount of $\mathrm{NaCl}$ equalled that of the hypotonic solution. Upon exchanging the hypotonic solution with the isotonic low $\mathrm{Na}$ solution a similar rapid fall in the active tension was observed (not illustrated). Thus, the rapid tension fall in phase 4 is probably not due to a negative inotropic effect of $\mathrm{Na}$ ions but rather to an abrupt change in the tonicity of solution, i.e. a relative hypertonicity in this case. 


\section{Measurement of muscle weight during hyper- and hypotonic perfusions}

If the ventricular muscle of bullfrog behaves as a simple osmometer the muscle volume would change in the reverse direction and degree with the tonicity of the perfusate. In order to avoid undesirable errors in measuring the tissue dimensions we measured the muscle weight rather than the volume. The muscle preparation was perfused with constant flow as the usual experiments, but was not driven. The preparation was taken up from the perfusing chamber every 5 minutes. Water was wiped up with a filtration paper and the muscle weight was measured as rapid as possible.

Figure 11A and B show the time course of change in the muscle weight caused
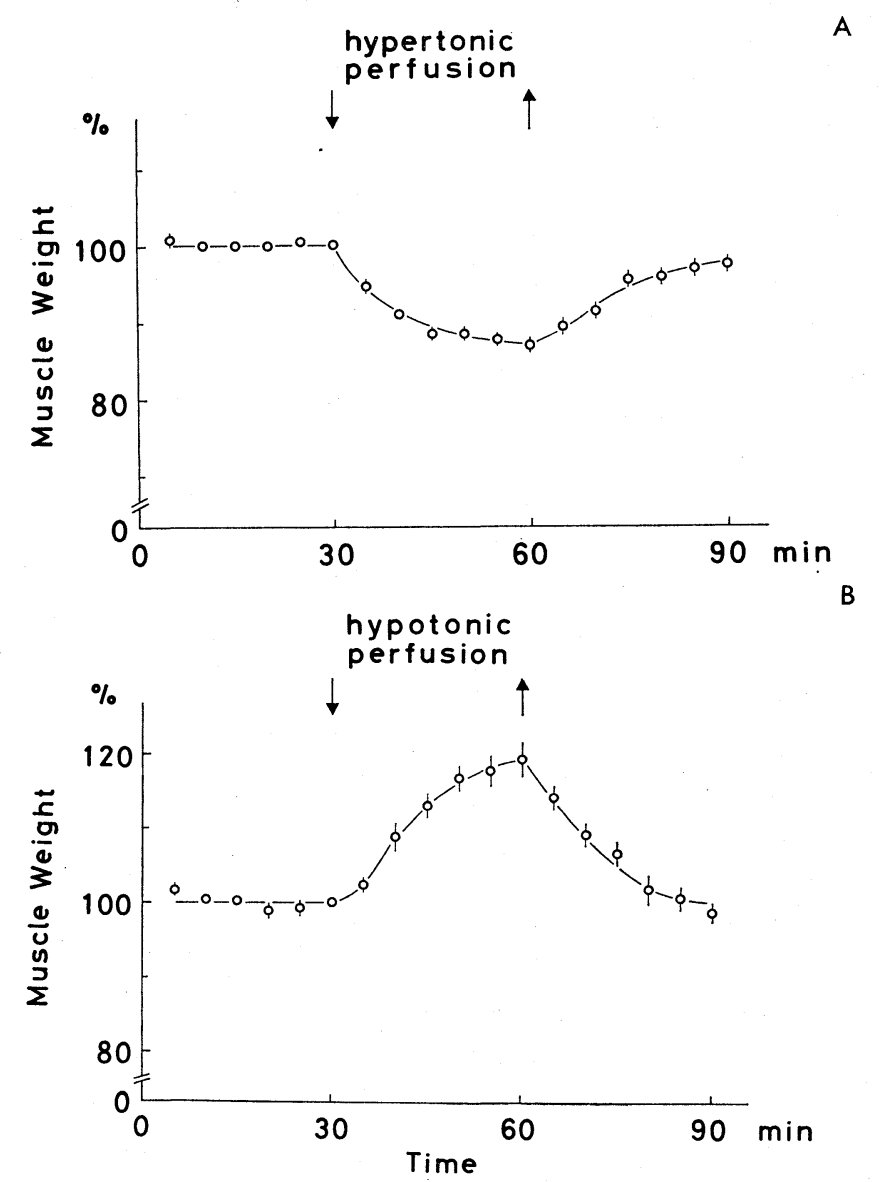

Fig. 11. Weight changes of bullfrog ventricle in hyper- and hypotonic perfusions. In A, between two arrows, hypertonic perfusion (tonicity $200 \%$ ) was applied for $30 \mathrm{~min}$. In $\mathrm{B}$ effect of the hypotonic (tonicity $50 \%$ ) solution is shown. Vertical bars indicate $\pm \mathrm{SE}$ of the mean. The control as well as the hyper- or hypotonic solutions were buffered with $2 \mathrm{mM}$ Tris- $\mathrm{HCl}$ and $\mathrm{NaHCO}_{3}$ and $\mathrm{KH}_{2} \mathrm{PO}_{4}$ were omitted. 
by perfusion with hypertonic $(200 \%)$ and hypotonic $(50 \%)$ solutions, respectively. Figure 11A summarizes the effect of hypertonicity obtained from five different muscles (mean muscle weight $11.1 \pm 0.99 \mathrm{mg}$ ). In the figure, between the two arrows, $\mathrm{NaCl}$-hypertonic solution was perfused for $30 \mathrm{~min}$. It is seen that the muscle weight begins to decrease with onset of hypertonic perfusion in a simple exponential manner and that it does not reach a final steady level even $30 \mathrm{~min}$ after the onset of perfusion. The recovery process requires a far longer time. It can be seen in the figure that a 30 min-perfusion with normal Ringer solution is not sufficient for restoration of the control muscle weight (compare with Fig. 2 and Fig. 7). There was no fundamental difference in the weight change between $\mathrm{NaCl}$ hypertonicity and sucrose hypertonicity (not shown).

Figure 11B shows the results obtained from another five muscles (mean muscle weight $8.32 \pm 0.69 \mathrm{mg}$ ). It is seen that in the hypotonic medium the muscle gains water with a somewhat different time course from the onset pattern of the hypertonic perfusion. The rising phase shows a rather S-shaped curve and the recovery phase exhibits a more rapid change than that observed in the hypertonicity. We have also examined the effects of $300 \%$-hypertonicity, $33.3 \%$ - and $12.5 \%$ hypotonicities on the muscle weight and obtained the essentially similar time courses. Thus, comparing with the skeletal muscle, the bullfrog heart muscle seems to have a considerable barrier for water movement though it consists of relatively loose, spongy structures.

\section{DISCUSSION}

The present study demonstrated that the alteration in the osmolarity of a perfusate affects the action potential as well as the contractile tension in a complicated way. The main findings are that the contractility is inhibited both by hyper- and hypotonic solutions and that the sustained contracture can be induced by perfusion of both solutions.

The pattern of the change in twitch contraction is of special interest since it strongly depended on the kind of hypertonic solutions. The rate of rise of contraction did not change in the sucrose hypertonicity. Contrary to our observations, KOCH-WESER (1963) reported that in the cat heart the change of peak tension caused by hypertonicity (sucrose or mannitol) is mainly due to change in the rate of tension development. LitTle and Sleator (1969), on the other hand, found that a positive inotropic effect was caused by raffinose hypertonicity in the guineapig atria and that this was accompanied with a significant decrease in the action potential duration. In the present investigation we found that the negative inotropism caused by sucrose hypertonicity is primarily attributed to the shortening of the action potential duration, whereas that caused by $\mathrm{NaCl}$ hypertonicity is due to some inhibitory mechanisms on the contractile process. The change in the electrical phenomena was not a major factor in the latter case. Thus, the 
relation between the change in the action potential and the pattern of the twitch inhibition seems not to be a simple one.

The speed of the plateau shortening markedly differed between the sucrose and the $\mathrm{NaCl}$ hypertonicities although there was no fundamental difference in the time course of the muscle shrinkage between both conditions. In the $\mathrm{NaCl}$ hypertonic solution the plateau shortening occurred far more slowly than in the sucrose-hypertonic solution. This slowness would be related to some antagonistic action of $\mathrm{Na}$ ions at the membrane site which directly controls the plateau mechanism.

The mechanism for the plateau shortening remains unknown. It is unlikely that changes in the membrane rectifier systems (instantaneous as well as delayed) are the main cause since we could not find any difference in the current-voltage relationship measured by the constant current method between the iso- and hypertonic conditions (unpublished observations). It is also unlikely that the entry of activator calcium, which is thought to directly contribute to the plateau phase (Niedergerke and OrKand, 1966; Hagiwara and NAKAJima, 1966), was decreased by hypertonic solution because the rate of force development remained practically unchanged during the hypertonic perfusion (Figs. 1A and 3b). However, if the major part of a single twitch of the bullfrog ventricle is not determined by a direct inflow of Ca ions, as postulated for mammalian myocardium (MASCHER, 1971; New and Trautwein, 1972; LANGER, 1973), the rate of force development would not necessarily decrease.

Recently HermsmeYer et al. (1972) proposed the hypothesis that the plateau loss seen during hypertonic perfusion is related to the swelling of the $\mathrm{T}$ system. The plateau of the action potential from animals which have the T-tubule system can be shortened by hypertonic solutions whereas that from animals such as frog or chicken which lack the $\mathrm{T}$ system cannot be altered by the same procedure. We cannot explain the discrepancy between their results and present findings since they also used hypertonic solutions (tonicity $200 \%$ ) prepared by addition of sucrose or $\mathrm{NaCl}$ and the experimental condition was essentially the same. The only difference would be in the species of frog used in these two investigations.

On the other hand, the changes in twitch contraction observed during $\mathrm{NaCl}$ hypertonic as well as hypotonic perfusion (Figs. 7 and 8) are of special interest. The contractile force declined without accompanying any shortening of time to peak tension during $\mathrm{NaCl}$-hypertonic perfusion. This inhibition in the twitch tension occurred with two different time constants, a very rapid one and a solwer one. The initial rapid fall cannot be attributed solely to the suppressive effect of excess $\mathrm{Na}$ in the outer medium but rather to the dehydration process due to hypertonicity since this inhibition occurred too rapid to be ascribed to $\mathrm{Na}$ effect.

Moreover, the fact that the rate constant of the rapid decreasing phase $\left(0.50 \mathrm{~min}^{-1}\right)$ in the $\mathrm{NaCl}$ hypertonicity was even larger than that of the plateaudependent falling phase $\left(0.40 \mathrm{~min}^{-1}\right)$ in the sucrose hypertonicity indicate that the 
effect is superficial, presumably in the vicinity of the muscle membrane. It may be possible to assume that there is a special superficial area which is critical for release and/or inflow of activator $\mathrm{Ca}$ and is very sensitive to the change in osmolarity. The existence of such a juxtamembranous superficial $\mathrm{Ca}$ compartment (like subsarcolemmal cisternae) had been repeatedly emphasized in the recent investigations on the E-C coupling process in the heart muscle (SANBORN and LANGER, 1970; Bassingswaighte and Reuter, 1972; Page and Niedergerke, 1972; LANGER, 1973). On the other hand, we cannot explain the reason why the rate of tension development was not suppressed in the sucrose hypertonicity. The slower phase of the twitch decline would result from the effect of hypertonicity to the deeper part of the fiber, i.e. the increase in the ionic strength around the contractile filaments since the time course of this slower phase roughly corresponds to that of the dehydration process. Recently LANGER (1973) reported that the initial depression in myocardial force which occurs during hyper-osmolar $[\mathrm{Na}]_{\text {o }}$ perfusion is followed by a secondary increase and that there is a force overshoot upon restitution of control $[\mathrm{Na}]_{0}$. On the basis of this finding he stressed the role of the $[\mathrm{Na}]_{i}$-dependent $\mathrm{Na}-\mathrm{Ca}$ exchange system. In the present investigation on the twitch contraction of frog ventricle we could not observe such a recovery phenomenon. Instead, we found the slowly decreasing phase of the active tension during hypertonic perfusion mentioned above.

As to the effects of hypotonic solution our experiments showed that there are at least three phases in the changes in twitch tension during hypotonic as well as recovery perfusion (Fig. 8). This may also suggest, together with the fact that neither the action potential nor the muscle weight showed any complicated time course, the possibility of the existence of a two-compartment system in the bullfrog ventricular muscle. The initial uprise (phase 1) in the tension can be explained by an effect of reduced $[\mathrm{Na}]_{0}$, although the extent of this phase considerably varied from preparation to preparation. The subsequent two phases may reflect the osmolarity effect on the two intracellular compartments.

It should be noted that there seems to exist some kind of rectification in the time course of the tension change when the tonicity of the bathing medium is altered. In general, the tension change occurred more rapidly when the tonicity was raised (absolutely or relatively )than when the latter was lowered. Thus, as shown in Figs. 2, 7, and 8, the twitch tension falls very rapidly when water flows out of the muscle although no such a marked asymmetry or rectifying phenomenon could be observed in the change of the muscle weight.

Recently, LäNNERGREN and NOTH (1973) reported that in the single fiber of frog skeletal muscle the hypertonic solutions above $150 \%$ or $200 \%$ of normal can elicit a phasic contraction which is superposed on a very small sustained tension increase. We could not find any such a type of transient contraction either in hyper- or hypotonic media. Instead, we found that contractures of a considerable amount can be produced in both osmolar conditions. The nature and the 
mechanism of these contractures remain to be explored further.

\section{REFERENCES}

Bassingthwaighte, J B. and Reuter, H. (1972) Calcium movements and excitation-contraction coupling in cardiac cells. In Electrical Phenomena in the Heart, ed. by De MeLlo, W. C. Academic Press, New York and London.

Goto, M. and Brooks, C. McC. (1969) Membrane excitability of the frog ventricle examined by long pulses. Am. J. Physiol., 217: 1236-1245.

Hagrwara, S. and Nakajima, S. (1966) Differences in Na and Ca spikes as examined by application of tetrodotoxin, procaine and manganese ions. J. Gen. Physiol., 49: 793-806.

Hermsmeyer, K., Rulton, R., and Sperelakis, N. (1972) Loss of the plateau of the cardiac action potential in hypertonic solutions. J. Gen. Physiol., 59: 779-793.

Hodgkin, A. L. and Horowicz, P. (1957) The differential action of hypertonic solution on the twitch and action potential of a muscle fiber. J. Physiol. (Lond.), 136: 17P.

Howarth, J. V. (1958) The behaviour of frog muscle in hypertonic solutions. J. Physiol. (Lond.), 114: 167-175.

IsAACSON, A. (1969) Caffeine-induced contractures and related calcium movements of muscle in hypertonic media. Experientia, 25: 1263-1265.

KaWATA, H. (1961) The influence of hypertonic solutions on the intracellular potential of frog ventricle. Fukuoka Acta Medica, 52: 635-647 (in Japanese).

KawatA, H. and Chen, Y. F. (1972) Post-anodal inhibition of the electrically induced sustained contraction in the skeletal muscle. Jap. J. Physiol., 22: 187-204.

КоCH-WESER, J. (1963) Influence of osmolarity of perfusate on contractility of mammalian myocardium. Am. J. Physiol., 204: 957-962.

LANGer, G. A. (1973) Excitation-contraction coupling. Ann. Rev. Physiol., 35: 55-86.

LÄNNERGREN, J. and NoTH, J. (1973) Tension in isolated frog muscle fibers induced by hypertonic solutions. J. Gen. Physiol., 61: 158-175.

LitTle, G. R. and Sleator,W. W. (1969) Calcium exchange and contraction strength of guineapig atrium in normal and hypertonic media. J. Gen. Physiol., 54: 494-511.

MASCHER, D. (1971) Electrical and mechanical events in depolarized cardiac muscle during low sodium perfusion. Pflügers Arch., 323: 284-296.

New, W. and Trautwein, W. (1972) Ionic nature of slow inward current and its relation to contraction. Pflügers Arch., 334: 24-38.

Niedergerke, R. and ORKand, R. K. (1966) The dual effect of calcium on the action potential of the frog's heart. J. Physiol. (Lond.), 184: 291-311.

PAGe, S. G. and Niedergerke, R. (1972) Structures of physiological interest in the frog heart ventricle. J. Cell Sci., 11: 179-203.

SAnborn, W. G. and LANGer, G. A. (1970) Specific uncoupling of excitation and contraction in mammalian cardiac tissue by lanthanum. J. Gen. Physiol., 56: 191-217.

Sperelakis, N., Hoshiko, T., Keller, R. F., Jr., and Berne, R. M. (1960) Intracellular and external recording from frog ventricular fibers during hypertonic perfusion. Am. J. Physiol., 198: $135-140$. 